\title{
La Importancia de los Determinantes Colectivos del Desempeño en la Evaluación de Competencias
}

\section{Competence Assesment: The Role of Collective Determinants of Performance}

\author{
Juan Andrés Pucheu ${ }^{1}$
}

\begin{abstract}
Resumen:
Hoy en día es posible observar el uso de múltiples modelos de competencias, la mayoría de los cuales asume que la capacidad o competencia es una propiedad del individuo y que este la puede ejercer con independencia de factores colectivos $u$ organizacionales. Esta falta de consideración por los determinantes colectivos o ambientales del desempeño es una debilidad que no sólo no corresponde al estado actual de las teorías científicas sobre personalidad, si no que además puede originar aplicaciones infructuosas e incluso la asignación inadecuada de responsabilidades ante deficiencias en el desempeño. Este artículo presenta una modelo de análisis que remedia estas carencias y señala dos ejemplos de aplicación obtenidos en estudios de consultoría e investigación académica.
\end{abstract}

\begin{abstract}
:
Currently there are many theoretical approaches and taxonomies about competencies. Regrettably, most competence models pay little or no attention to collective performance determinants and physical conditions. Current personality theory does not allow this omission, which could result in irrelevant interventions or, even worse, inadequate distribution of responsibilities. This paper presents a model of analysis that corrects this failure and two examples of its application obtained from consulting and academic research experiences.
\end{abstract}

${ }^{1}$ Psicólogo organizacional, master of business administration, doctorando en psicología. Pontificia Universidad Católica de Chile y Universidad de Santiago de Chile. Para comentarios o contactos en relación con este artículo escribir a apucheu@uc.cl. 
La evaluación del desempeño y los factores que lo determinan es uno de los temas centrales en gestión y psicología organizacional. En los últimos decenios este tema ha sido abordado en la práctica de consultoría principalmente desde la representación social de Gestión por Competencias, la cual ha llegado a ser una de las herramientas de administración y consultoría más aplicadas en estos momentos (Rodríguez et al., 2002). Lamentablemente, el hecho de que muchos modelos de competencias carezcan de un substrato teórico coherente ha dificultado tanto la evaluación de su validez como la convergencia hacia un sistema de aceptación universal. Una característica particularmente riesgosa de los modelos de competencias es el supuesto implícito de que el desempeño es determinado total o fundamentalmente por características individuales, lo que obvia consideraciones sobre capacidades organizacionales e incluso las condiciones físicas ambientales requeridas para el buen desempeño. Esta debilidad puede resultar en implementaciones de gestión por competencias inadecuadas o el uso de la evaluación de competencias como una forma errónea de asignación de responsabilidades en el análisis de fallas en procesos de trabajo. Considerando lo anterior, el objetivo central de este artículo es precisar el concepto técnico de competencia y sus limitaciones, sugiriendo adicionalmente algunas mejoras que pueden ser efectuadas a partir de más precisas y válidas definiciones de sus determinantes. El fin último de este esfuerzo sería una mayor eficacia en la gestión del desempeño y los procesos de desarrollo organizacional en los sectores público y privado.

\section{El contexto de cambio organizacional en el que se aplica la gestión por competencias}

Comenzaremos este artículo reseñando brevemente el contexto de cambio tecnológico y de estructuras organizacionales que ha permitido la difusión de la idea de gestión por competencias. Para lo cual es necesario partir por recordar que el desarrollo de sistemas de redes informáticas ha posibilitado, desde fines de los años setenta, la progresiva automatización de los procesos de trabajo y la consiguiente eliminación de las actividades humanas que estaban implicadas en estos procesos. La primera oleada de automatización, comúnmente asociada a la idea de reingeniería, consistió en la automatización de procesos administrativos y productivos de alta estabilidad y recurrencia, tales como sistemas administrativo-contables y líneas de montaje. Esto implicó que las actividades asociadas al trabajo humano quedaran reducidas a dos grandes tipos de procesos. El primer tipo incluye a aquellas actividades cuya falta de definición o variabilidad las hace dependientes de aprendizajes procedurales o códigos sociales implícitos, tales como las habilidades sociales asociadas a venta, atención de público, supervisión, enseñanza o negociación. En segundo lugar están los procesos cuya complejidad o carácter adhocrático hace necesaria la toma de decisiones complejas o la creatividad, tales como la generación de estrategias o el mismo diseño de procedimientos. En la actualidad el aumento en la capacidad de procesamiento de los sistemas informáticos y la difusión de prácticas de auto servicio y compra a través de Internet, así como los sistemas de control de gestión informatizados, entre otras tecnologías, están permitiendo que el trabajo asociado a habilidades sociales también este siendo reemplazado, al menos parcialmente, por informatización. De esta manera esta quedando como trabajo humano algunas actividades físicas de bajo costo o no recurrentes $\mathrm{y}$, en el extremo de mayor valor, actividades de integración creativa o toma de decisiones complejas. Si bien esta disminución de los ámbitos tradicionales de trabajo humano se encuentra todavía en evolución y en muchos casos es parcial o no económicamente conveniente para las empresas, ya ha manifestado una gran influencia en el tamaño y tipo de estructura de muchas empresas y gran parte del Estado. 
Desde el punto de vista del volumen de trabajo se puede señalar que el uso intensivo de sistemas informatizados y el acceso a Internet permiten crecimientos casi instantáneos en el número de puntos de venta, generación e intercambio de información en dimensiones imposibles de lograr a través del contacto humano y, vía Extra e Intranet, el funcionamiento integrado de múltiples proveedores, los cuales pueden combinar las fortalezas de sus imágenes corporativas y redes de clientes (Evans \& Wurster, 2000). Un ejemplo evidente de estos fenómenos es la aparición de canales de distribución masiva que compiten entre sí a pesar de provenir de industrias originalmente tan diferentes como la banca, supermercados, vestuario o ferretería.

Desde el punto de vista del cambio observable en las estructuras organizacionales es posible diferenciar tres niveles de análisis: Cambios en la representación de las estructuras, cambios en la combinación de mecanismos de coordinación de procesos y, finalmente, cambios en los contenidos y complejidad de las identidades asumidas por las personas implicadas en la organización. En la representación organizacional observamos el paso desde un énfasis interno concreto a un énfasis externo y de mayor abstracción, lo que corresponde respectivamente a la evolución desde representaciones organizadas en función de organigramas y descripciones de cargo agrupadas en función de su similitud a representaciones de las actividades agrupadas secuencialmente en función de su impacto en la decisión de compra o evaluación del cliente. Ejemplificando lo anterior se puede señalar que hemos pasado desde un tipo de estructura en la cual todos los contadores se encontraban en el departamento de contabilidad y todos los vendedores en el de ventas a una en la cual la unidad de análisis es la actividad, las cuales deben organizarse intercalando automatización y acción humana. Este cambio desde la unidad de análisis puesto de trabajo a la unidad de análisis actividad ha sido posibilitado por la apa- rición de toda una gama de modelos y productos, entre los que se incluyen desde la descripción de procesos implicadas en los ERP hasta modelos contables como ABC.

En el nivel de análisis de los mecanismos de coordinación y utilizando la taxonomía de Mintzberg (Ver: Mintzberg \& Quinn, 1993), se puede señalar que ha sido posible el cambio desde burocracias mecánicas a burocracias profesionales. El primer tipo, la burocracia mecánica, alude a organizaciones que dependen, para la estabilidad de su funcionamiento, tanto de la estandarización de procesos lograda a través de formularios y reglamentos como de la gestión de excepciones efectuada por la casta de supervisores de nivel medio, los cuales constituyen como grupo un mecanismo con la capacidad logística y los criterios necesarios para integrar reglamentos incongruentes o administrar la innovación. Esta estructura es adecuada para ambientes estables y producción de bienes y servicios estandarizados, lo que reduce la complejidad lo suficiente como para permitir la existencia de controles centralizados efectivos. La burocracia profesional, en cambio, se caracterizaría su adecuación a entornos más flexibles y segmentados, lo que se posibilita por el uso de una combinación de tres tipos de mecanismos de coordinación que deben actuar integradamente: La estandarización de criterios, la distribución de información en la forma de sistemas de control de gestión y, en tercer lugar, estandarización de capacidades. Muchas de las herramientas de gestión actualmente en desarrollo o aplicación, incluyendo la gestión por competencias y varias de las representaciones relacionadas, como mejores prácticas, sistemas de control de gestión, benchmarking o gestión del conocimiento, pueden considerarse intentos de estandarización o control de criterios, información o capacidades, que son los mecanismos de coordinación necesarios cuando los individuos deben gestionar procesos flexibles en condiciones cambiantes y sin el beneficio de experiencia o una 
dirección centralizada con la suficiente capacidad de análisis para adecuar sus decisiones a cada situación particular.

Finalmente, en lo que corresponde al nivel de análisis del cambio en las identidades es observable un aumento en la complejidad y abstracción de las identidades asociadas a los roles organizacionales. De esta manera las identidades asociadas a jerarquía, clase, edad, género y educación formal pierden importancia relativa con relación a la generada por el aporte a la organización, el cual es establecido a través de sistemas de acreditación de capacidades. Desde este punto de vista la acreditación de competencias puede ser considerada un sistema de construcción y validación de la identidad que compite con los discursos culturales tradicionales, que apoyaban el funcionamiento basado en estandarización de procesos y la generación de redes sociales que, bajo la denominación de equipos o comités de supervisión, permitían la evaluación y gestión de excepciones.

\section{Los modelos de gestión por competencias}

En nuestro medio los cultores de la gestión por competencias suelen atribuir a D. McClelland la paternidad de la idea de competencia y la concepción de que el desempeño es multideterminado. Esto debido a su artículo Testing for competence rather than intelligence, de 1973 (Spencer et al., 1994). Estas creencias son cuestionables en función de tres observaciones: La primera es que es posible observar críticas a los modelos de explicación del rendimiento basados en un sólo factor, ya sea motivación, inteligencia o personalidad, casi desde el mismo momento que fueron formulados (Nash, 1992). La segunda es que es posible encontrar equivalentes del actual concepto de competencia aplicada al análisis de puestos de trabajo tan lejos como 1913 (Leplat, 2001) y la tercera es que, de hecho, el concepto competencias fue utilizado en primer lugar por R. White, en su artículo Motivation reconsidered: the concept of competence, aparecido en la revista Psychological Review en 1959 (Delamare \& Winterton, 2005).

En estos momentos es posible discriminar cuatro orientaciones principales en la aplicación de la Gestión por Competencias: La funcionalista, la conductista, la constructivista y, más recientemente, los llamados modelos integrativos. La corriente funcionalista, originada en la revisión de los sistemas de formación técnica ingleses a finales de la década de los setenta y comienzos de los ochenta, se orienta a identificar las capacidades organizacionales claves a partir de la observación de las cadenas de valor. En la práctica se intentan definir capacidades asociadas a los procesos de trabajo, las cuales posteriormente son agrupadas para optimizar su entrenamiento. La conductista, posiblemente la línea de pensamiento más difundida en nuestro medio, se caracterizaría por orientarse hacia la búsqueda de determinantes de desempeño individual utilizando, para su estudio, a sujetos que hayan alcanzado desempeños sobresalientes. Ahora bien, en la práctica su aplicación se realiza a través de la adaptación de diccionarios de competencias, los cuales se han ido originando en la práctica de las empresas consultoras. La corriente constructivista implica un enfoque de aprendizaje a través de procesos de integración de los discursos declarados por todos los miembros de una organización, incluyendo en esto a trabajadores con distintos niveles de rendimiento. Finalmente, es necesario señalar que últimamente han aparecido modelos que reclaman la calidad de integrativos y que, efectivamente, presentan características u orientaciones mixtas que superponen los modelos anteriores de manera más o menos sistemática. Dejando de lado la necesidad comercial de diferenciarse de las empresas consultoras que proponen modelos integrativos, parece razonable considerar su aparición debido a la aparente complementariedad de las tres corrientes originales. Así parece lógico señalar que un aná- 
lisis de capacidades debe comenzar por definir los procesos y capacidades críticas para el cumplimiento de la estrategia, tal como señala el enfoque funcionalista. Luego estas capacidades deben ser analizadas en sus componentes, tal como aporta el enfoque conductual $\mathrm{y}$, en el momento de la implementación, no es posible obviar la necesidad de generar una representación consensuada de cada capacidad y sus estándares, lo que constituye la especialidad del aporte constructivista.

\section{Competencia como capacidad y sus determinantes}

Dada la gran cantidad de modelos de competencia ofrecidos por la empresas consultoras y la falta de un relación fluida entre la práctica de gestión de recursos humanos y la academia (Burke et al., 2004), en estos momentos no contamos con un concepto universalmente aceptado de competencia. Pese a esto es posible observar que existe un énfasis en la capacidad individual por sobre las capacidades colectivas al mismo tiempo que la creencia de que la competencia es la manifestación de cualidades subyacentes que la determinan (Pucheu, 2002). Lamentablemente, al intentar analizar las competencias a partir de factores cuyas definiciones se encuentran en diversos niveles de agregación o desarrollo, se producen superposiciones que dificultan aun más el logro de homologaciones entre los modelos. Por ejemplo, McClelland originalmente distinguió cinco tipos de determinantes, todos considerables como cualidades individuales: Motivos, rasgos, auto conceptos, conocimientos y destrezas (Spencer \& McClelland, 1990). Si bien el uso de estas categorías podría haber sido adecuada a comienzos de los setenta, un periodo de renovación conceptual en psicología, hoy debería ser cuestionada. Por ejemplo, la distinción entre motivos y rasgos podría ser cuestionada basándose en la evidencia de su relación en tanto orientadores de conducta (Ejemplos:
Capozza \& Brown, 2000; Hurtz, \& Donovan, 2000; Roberts \& Robins, 2000). Así también, la distinción entre rasgos y auto concepto cuenta con límites difusos (Ver: Cross et al., 2002; Fleeson, 2001), lo mismo que la de motivos y autoconceptos (Ejemplos: Duncan \& Agronick, 1995; Vancouver et al., 2001) y la de destrezas y motivación (Ejemplos: Kanfer \& Ackerman, 1989; Vitkus \& Horowitz, 1987).

Para superar los problemas generados por la superposición conceptual es necesario utilizar dos criterios mínimos en la elección de los constructos determinantes de la capacidad o competencia: El primero es utilizar modelos de uso aceptado en los ámbitos de la psicología científica y la administración, el segundo, es utilizar categorizaciones mutuamente excluyentes. Para efectos del análisis de capacidades individuales ambos criterios son cumplidos al utilizar las categorías correspondientes a los constructos de conocimiento, habilidad, valores \& creencias, características de personalidad e información (Pucheu, 2002). En esta elección estamos siguiendo principalmente la línea de pensamiento de McCloy, Campbell \& Cudeck (1994), quienes identificaron 3 factores individuales que determinan el desempeño: Conocimiento declarativo, conocimiento procedural y habilidades y, en tercer lugar, la elección motivacional. Posteriores publicaciones (Baum \& Locke, 2004; Hurtz \& Donovan, 2000; Salgado, 1997; Vinchur, Schippmann, Switzer \& Roth, 1998), han permitido añadir a la taxonomía de factores determinantes la categoría de rasgos o atribuciones sobre la personalidad. Adicionalmente, a los cuatro factores anteriores es necesario agregar la categoría de información, la cual si bien es una cualidad organizacional, está siendo ampliamente estudiada en función del aumento de la complejidad y plasticidad de los contextos organizacionales (Bobillier, 2003; Seely \& Duguid, 2000; Becker et al., 2001). 
Es importante señalar que la concepción de rasgos o características personales ha evolucionado de tal manera que ahora se incluyen, además de las tendencias de base biológica señaladas en los 5 grandes, atribuciones personales asociadas a nivel sociocultural, género, edad o etapa de vida, acreditación de educación formal, origen nacional e incluso antigüedad en la organización, además de otras consideraciones idiosincrásicas a la base de establecimiento de confianza o validación personal. Estas categorías permiten establecer una relación sustantiva entre la cultura organizacional y las capacidades individuales aún ante similitud en las tecnologías, los ambientes físicos u otras características organizacionales. Siendo además teóricamente compatibles con modelos post estructuralistas de la personalidad, la motivación y las relaciones de liderazgo (Ejemplos: Paunonen \& Jackson, 2000; Balkundi \& Kilduff, 2006; Reicher et al., 2005).

Adicionalmente, el uso de constructos mutuamente excluyentes y en uso cuenta con la ventaja de que es posible ligarlos directamente a áreas específicas dentro de la gestión de recursos humanos. En efecto, el conocimiento declarativo puede ser adquirido por capacitación o especialización técnico profesional; las habilidades pueden ser desarrolladas a través de entrenamiento o evaluadas en la selección de personal; los valores y creencias pueden intervenirse a través de prácticas de inducción, liderazgo y comunicaciones; las características de personalidad son el objeto de coaching y selección y, en quinto y último lugar, la información es una cualidad organizaciones objeto de herramientas como planificación, sistemas de control de gestión y comunicaciones internas.

\section{Ejemplos de aplicación}

La aplicación de la taxonomía propuesta de determinantes de la capacidad ha permitido implementar diversos modelos de gestión de competencias en empresas naciona- les. Esto posibilita la muestra de ejemplos en los casos de competencias claves para roles de supervisión y ventas.

En el caso de competencias de supervisión podemos mencionar, como ejemplo, la capacidad de influir en la conducta voluntaria de los sus subordinados, que es clave no sólo en diversos modelos de supervisión y liderazgo, sino que además ha sido identificada como una de tres capacidades claves el análisis de competencias realizado en una empresa financiera nacional. El estudio realizado en esta empresa durante el año 2004 permitió también identificar determinantes en las cinco categorías antes descritas. La tabla 1 muestra estos determinantes. 
Tabla 1: Determi nantes de la capacidad de Influir en la conducta voluntaria de subordinados y sistemas de gestión del desempeño asociados a cada determi nante

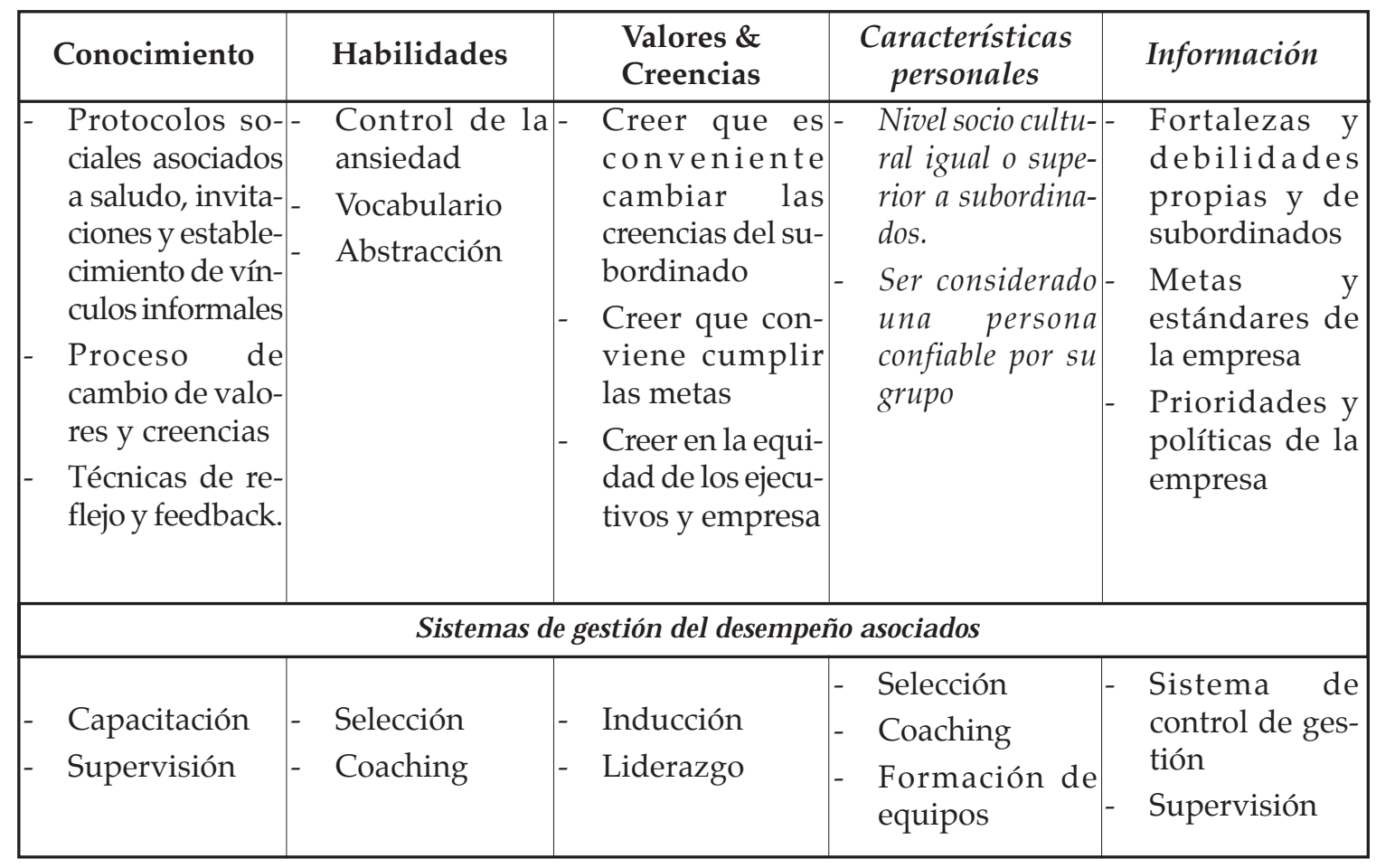

Como se puede observar en la tabla anterior, elementos como la atribución de ser una persona confiable o un nivel socio cultural similar o superior al de los subordinados son características claves para poder implementar exitosamente las acciones que permiten influir sobre los subordinados. Dado que estas características no son generalizables a distintas situaciones y dependen de la historia e interpretación que las distintas partes hacen de la relación, deben considerarse propiedades de la relación entre supervisor y subalterno y no de este último. En lo que toca con al conocimiento de las fortalezas y debilidades de cada subalterno, lo cual es posible de evaluar en función de las metas, políticas, estándares y prioridades de la empresa, información que es adquirida de manera informal en gran parte de las empresas chilenas, nos encontramos también con determinantes de la capacidad que no es posible de generalizar de una a otra situación. Estos ejemplos confirman la necesidad de considerar a las capacidades no sólo como una propiedad individual, si no que organizacional. Esto también es valido para otras capacidades críticas en supervisión, tales como planificar el trabajo del grupo de subordinados u obtener apoyo y recursos en caso de cambios o imprevistos, en las que incidencia de la calidad de las redes informales de la organización se hace aún más evidente.

Un segundo ejemplo que permite observar la incidencia de cualidades organizacionales es la competencia Manejar objeciones, la cual ha sido identificada como una de las capacidades claves en vendedores del área financiera en el análisis realizado por Burgos et al. (2005). Al igual que en el caso de la competencia de supervisión, este análisis se realizó en una empresa chilena del área financiera, la que si bien es distinta a la anterior, también se cuenta entre las de ma- 
yor participación de mercado. El análisis de los determinantes que predicen un desempeño superior en esta capacidad se presenta en la tabla 2.

Tabla 2: D eterminantes de desempeño superior en la capacidad de manejar objeciones y sistemas de gestión del desempeño asociados a cada determinante ${ }^{2}$.

\begin{tabular}{|c|c|c|c|c|}
\hline Conocimiento & Habilidades & $\begin{array}{l}\text { Valores \& } \\
\text { Creencias }\end{array}$ & $\begin{array}{c}\text { Características } \\
\text { personales }\end{array}$ & Información \\
\hline \begin{tabular}{|l}
$\begin{array}{l}\text { Proceso de la } \\
\text { entrevista de } \\
\text { ventas } \\
\text { - } \\
\text { Técnica de ma- } \\
\text { nejo de objecio- } \\
\text { nes } \\
\text { - Políticas y pro- } \\
\text { cedimientos } \\
\text { administrativos } \\
\text { - Matemáticas } \\
\text { básicas }\end{array}$ \\
\end{tabular} & 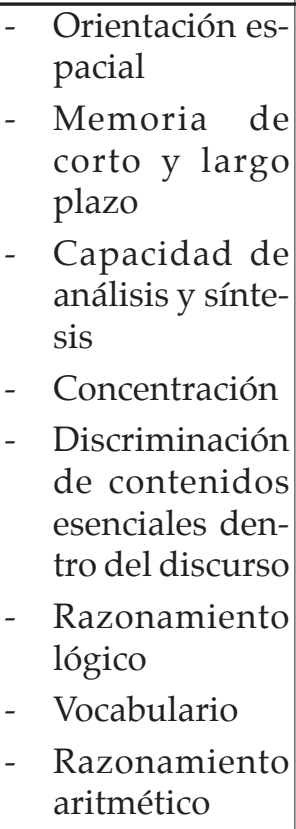 & $\begin{array}{l}\text { - "Si trabajo es } \\
\text { para cumplir } \\
\text { mis metas per- } \\
\text { sonales" } \\
\text { - "Me siento feliz } \\
\text { de trabajar en } \\
\text { esta empresa y } \\
\text { ser vendedor" } \\
\text { - "Para poder } \\
\text { vender se debe } \\
\text { ser transparente } \\
\text { y no enganar al } \\
\text { cliente" }\end{array}$ & 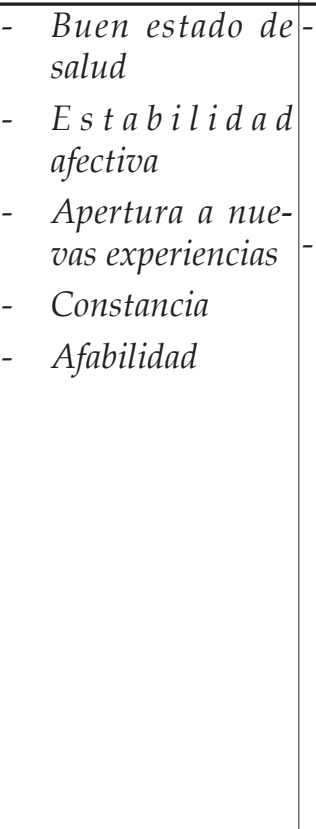 & $\begin{array}{l}\text { Características } \\
\text { de los productos } \\
\text { y sus similares } \\
\text { en empresas } \\
\text { competidoras } \\
\text { Ventajas y limi- } \\
\text { taciones de los } \\
\text { productos desde } \\
\text { el punto de vista } \\
\text { de los clientes o } \\
\text { segmentos obje- } \\
\text { tivos }\end{array}$ \\
\hline \multicolumn{5}{|c|}{ Sistemas de gestión del desempeño asociados } \\
\hline $\begin{array}{l}\text { - Capacitación } \\
\text { - Supervisión }\end{array}$ & - $\begin{array}{l}\text { Selección } \\
\text { - }\end{array}$ & $\begin{array}{ll}\text { - } & \text { Inducción } \\
\text { - } & \text { Liderazgo }\end{array}$ & 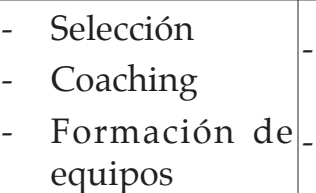 & $\begin{array}{l}\text { Sistema de con- } \\
\text { trol de gestión } \\
\text { Supervisión }\end{array}$ \\
\hline
\end{tabular}

Si bien la tabla 2 no presenta la totalidad de los determinantes, si no sólo aquellos que están asociados a un desempeño superior, es posible observar que factores como la estabilidad afectiva o el acceso a información sobre los competidores juegan un papel clave en la posibilidad de que un vendedor pueda manejar las objeciones ocasionadas por la desconfianza, falta de información e incluso comparación puntual entre cualidades del producto que hace el cliente. Aún más significativo que los factores anteriores es el hecho de que la motivación producida por creer en la bondad del producto y la empresa, así como la satisfacción con la ocupación de vendedor, definen atributos como persistencia o control de la ansiedad y, de esta manera, permiten observar la importancia clave de acti-

${ }^{2}$ Adaptado de Burgos et al., 2005. Página 67. 
vidades de inducción y las prácticas de liderazgo de supervisores y ejecutivos. Dado que estos factores son contextuales y pueden ser manejados, al menos parcialmente, a través de prácticas adecuadas de coaching, control de gestión y supervisión, este ejemplo reafirma la necesidad de considerar factores colectivos y organizacionales en el análisis de los determinantes de las competencias.

\section{Conclusiones}

El análisis y ejemplos presentados permite concluir básicamente tres ideas. La primera es que el uso de una taxonomía de determinantes de las competencias que contiene categorías mutuamente excluyentes facilita la identificación de carencias y ámbitos de acción correctiva. La segunda es que es necesario considerar que la información y algunas características personales, tales como atribuciones de confiabilidad o adecuación sociocultural, así como las creencias y valores que determinan la satisfacción con la identidad y expectativas de logro, son elementos que resultan de la interacción entre sujeto y organización, por lo que no pueden ser evaluados o acreditados de manera individual. Esto nos lleva a señalar, como tercera conclusión, que existe la imperiosa necesidad de cuestionar la aplicación de taxonomías de competencias y determinantes de competencias que se basen en teorías ya falseadas o concepciones del desempeño que no consideren la integración existente entre el sujeto y la organización. El no cuestionamiento de estos modelos implica asumir el riesgo de acreditar capacidades no generalizables o desestimar imprudentemente las responsabilidades e importancia de los distintos sistemas de gestión o apoyo al desempeño.

Si bien podría señalarse que el enfoque constructivista evita estos riesgos al generar clasificaciones de competencias desde la conversación de miembros de todos los niveles y ámbitos organizacionales, la concepción de competencia como cualidad individual pue- de influir en este proceso de la misma manera que lo hace en las adaptaciones de diccionarios o clasificaciones que son realizadas en los otros enfoques y por tanto, como idea final no queda sino recordar la conveniencia de un dialogo constante entre las prácticas de gestión de recursos humanos y el trabajo académico.

\section{Bibliografía}

BALKUndi, P. \& KiLdufF, M. (2006) The ties that lead: a social network approach to leadership. The Leadership Quarterly, 16, 941-961.

BAUm, J. \& LoCKe, E. (2004) The relationship of entrepreneurial traits, skill, and motivation to subsequent venture growth. Journal of Applied Psychology, 89, 587-598.

BecKer, B., Huselid, M. \& Ulrich, D. (2001) El cuadro de mando de RRHH, vinculando las personas, la estrategia y el rendimiento de la empresa. Barcelona: Gestión 2000.

BobilLIER, M.E. (2003) Evolutions techniques et mutations du travail: Emergence de nouveaux modèles d'activité. Le Travail Humain, 66, 163-194.

Burgos, P., Cabezas, A. \& Ibarra, L. (2005) Aplicación de un profesiograma para determinar las areas capacitables en la fuerza de ventas de tarjeta de crédito del Banco Santander Banefe. Tesis presentada como parte de los requisitos de la obtención del título de psicólogo, con mención social y de las organizaciones. Escuela de Psicología de la Universidad de Santiago de Chile.

Burke, M., Drasgow, F., Edwards, J. (2004) Closing science-practice knowledge gaps: Contributions of psychological research to human resource management. Human Resource Management, 43, 299-304.

Cross, S., Morris, M. \& Gore, J. (2002) Thinking about oneself and others: the 
relational-interdependent self-construal and social cognition. Journal of Personality and Social Psychology. 82, 399-418.

Delamare, F. \& Winterton, J. (2005) What is competence? Human Resource Development International, 8, 27-46.

Duncan, L. \& Agronick, G. (1995) The intersection of life stage and social events: personality and life outcomes. Journal of Personality and Social Psychology, 69, 558-568.

Evans, P. \& Wurster, T. (2000). Blown to bits, how the new economics of information transforms strategy. Boston: Harvard Business School Press.

FleEson, W. (2001) Toward a structure- and process- integrated view of personality: traits as density distributions of states. Journal of Personality and Social Psychology. 80, 1011-1022.

Hurtz, G. \& Donovan, J. (2000) Personality and job performance: The big five revisited. Journal of Personality and Social Psychology. 85, 869-879.

KANFER, R. \& ACKERMAN, P. (1989) Motivation and cognitive abilities: an integrativaaptitude-treatment interaction approach to skill acquisition. Journal of Applied Psychology, 74, 657-690.

Leplat, J. (2001) La psychologie du travail, Psychologie Française, 45, 83-96.

McCloy, R., Campbell, J. \& Cudeck, R. (1994) A confirmatory model of performance determinants. Journal of Applied Psychology, 79,493-505

MinTZBerG, H.\& Quinn, J. (1993). El proceso estratégico. México: Prentice-Hall Hispanoamericana.

NASH, M. (1992) ¿Cómo incrementar la productividad del recurso humano?. Bogota: Norma.
PAunonen, S. \& JACKsON, D. (2000) What is beyond the big five? Plenty! Journal of Personality. 68, 821-835.

Pucheu, J. A. (2002) Algunas precisiones sobre la idea de competencia y sus componentes. Revista Chilena de Psicología, 23, 36-43.

Reicher, S., Haslam, S.A., \& Hopkins, N. (2005) Social identity and the dynamics of leadership: leaders and followers as collaborative agents in the transformation of social reality. The Leadership Quarterly, $16,547-568$.

Roberts, B. \& Robins, R. (2000) Broad dispositions, broad aspirations: the intersection of personality traits and major life goals. Personality and Social Psychology Bulletin. 26, 1284-1296.

Rodriguez, D,. Patel, R., Bright, A., Gregory, D. \& Gowing, M. (2002) Developing competency models to promote integrated human resource practices. Human Resource Management, 41, 309-324.

Salgado, J. (1997) The five factor model of personality and job performance in the European community. Journal of Applied Psychology, 82, 30-43.

Seely, J. \& Duguid, P. (2000) The social life of information. US: Harvard Business School Press.

SPencer, L. \& McClelland, D. (1990) A history and state of the art of job competency assessment methods. Artículo presentado en la conferencia anual de la American Psychological Asociation, Boston.

Spencer, L., McClelland, D. \& Spencer, S. (1994) Competency Assessment Methods, History and State of the Art. US: Hay / McBer Research Press.

Vinchur, A., Schippmann, J., Switzer, F. \& Roтн, P. (1998) A meta-analytic review of 
predictors of job performance for salespeople. Journal of Applied Psychology, $83,586-597$.

Vancouver, J., Thompson, C. \& Williams, A. (2001) The changing signs in the relationships among self-efficacy; personal goals, and performance. Journal of Applied Psychology. 86, 605-620.

VitKus, J. \& Horowitz, L. (1987) Poor social performance of lonely people: lacking a skill or adopting a role. Journal of Personality and Social Psychology, 52, 1266-1273.

Fecha Recepción Artículo: 2 de noviembre 2005 Fecha Evaluación Final: 22 de mayo 2006 
